\title{
Coupling Quantum States through a Continuum: A Mesoscopic Multistate Fano Resonance
}

\author{
Y. Yoon, ${ }^{1}$ M.-G. Kang, ${ }^{1}$ T. Morimoto, ${ }^{2}$ M. Kida,${ }^{3}$ N. Aoki, ${ }^{3}$ J. L. Reno, ${ }^{4}$ \\ Y. Ochiai, ${ }^{3}$ L. Mourokh, ${ }^{5}$ J. Fransson, ${ }^{6}$ and J. P. Bird ${ }^{1,3}$ \\ ${ }^{1}$ Department of Electrical Engineering, University at Buffalo, the State University of New York, Buffalo, New York 14260-1920, USA \\ ${ }^{2}$ Advanced Device Laboratory, RIKEN, 2-1 Hirosawa, Wako, Saitama 351-0198, Japan \\ ${ }^{3}$ Graduate School of Advanced Integration Science, Chiba University, 1-33 Yayoi-cho, Inage-ku, Chiba 263-8522, Japan \\ ${ }^{4}$ CINT Science Department, Sandia National Laboratories, P.O. Box 5800, Albuquerque, New Mexico 87185-1303, USA \\ ${ }^{5}$ Department of Physics, Queens College of CUNY, 65-30 Kissena Blvd., Flushing, New York 11367, USA \\ ${ }^{6}$ Department of Physics and Astronomy, Uppsala University, Box 530, SE-751 21 Uppsala, Sweden
}

(Received 6 May 2011; published 24 April 2012)

\begin{abstract}
We demonstrate a fully tunable realization of a multistate Fano resonance, in which a pair of remote quantum states experience an effective coupling due to their mutual overlap with a continuum. Our mesoscopic implementation of this system exploits the ability of the semiconductor nanostructures known as quantum point contacts (QPCs) to serve, in the low-density limit close to pinch-off, as an on-demand localized state. By coupling the states formed on two separate QPCs, through a two-dimensional electron gas that serves as a continuum, we observe a robust effective interaction between the QPCs. To explain this result, we develop a theoretical formulation, based on the ideas of the Schrieffer-Wolff transformation, which is able to reproduce our key experimental findings. According to this model, the robust character of the interaction between the two remote states arises from the fact that the interaction is essentially mediated by a large number of degenerate continuum states. While the continuum is often viewed as a source of decoherence, our experiment therefore instead suggests the possibility of using this medium to support the interaction of quantum states, a result that may allow new approaches to coherently couple nanostructures in extended geometries.
\end{abstract}

DOI: 10.1103/PhysRevX.2.021003

Subject Areas: Mesoscopics

\section{INTRODUCTION}

The interaction of discrete quantum objects allows for the emergence of complex phenomena in solid-state physics, with prominent examples provided by the interaction of localized and mobile spins in the Kondo effect and the phonon-mediated pairing of electrons in superconductivity. When the interaction arises from wave-function overlap among different atoms, the result is the emergence of covalent bonding that supports the formation of stable molecules and crystals. Yet another important form of interaction is that which arises widely in atomic physics: when the discrete levels of a particular atom are coupled to a continuum of unconstrained states. The role of this coupling was first revealed in studies of atomic autoionization, a process that arises from the interference of two separate channels for ionization [1,2]. One of these channels consists of the direct excitation of an electron to the continuum, while the second involves an intermediate, discrete state that is coupled to the continuum through a so-called configuration interaction [1]. The characteristic signature of the interference of these channels is the Fano resonance (FR) that is observed in scattering experiments,

Published by the American Physical Society under the terms of the Creative Commons Attribution 3.0 License. Further distribution of this work must maintain attribution to the author(s) and the published article's title, journal citation, and DOI. with a line shape that intimately reflects the relative transmission amplitudes of the two ionization pathways [1-9].

An important goal that has emerged in the field of mesoscopic physics is to mimic the interactions that arise in nature, as a means to extend the functionality of semiconductor nanodevices. In addition to the technological importance of this issue, this approach provides an invaluable way to explore quantum-mechanical interactions at the microscopic level, with a control that is simply not possible in conventional atomic systems. There have been numerous reports [10-18], for example, concerning the use of quantum dots as artificial atoms, providing a highly tunable system for investigations of new aspects of FR phenomenology (see the reviews of Refs. $[19,20]$ ). In other work, coupled quantum-dot molecules have been used to provide a controlled implementation of a two-level system, in which the strength of the interdot coupling is determined by the overlap of the decaying dot wave functions inside a common tunnel barrier. With each of these dots occupied by just a single electron, this overlap can be used to regulate the exchange interaction of the associated electron spins (as recently reviewed in Ref. [21]).

Building on the ideas developed in the works cited above, the objective of this work is to demonstrate how a robust interaction can be achieved between mesoscopic devices, by, perhaps counter intuitively, separating them from each other. In this approach, which utilizes the discrete-state/continuum coupling that is the hallmark of 
the FR, localized states are formed on two separate quantum dots and interact indirectly with each other through an interconnecting continuum. In spite of this nonlocal coupling, our experiments show evidence of a remarkably robust interaction between the two states, significantly larger than that which typically arises in quantum-dot molecules. Theoretically, we attribute this finding to the fact that each of the discrete states overlaps strongly with the states of the continuum, and that a large number of degenerate continuum states are involved in this interaction. This observation should be contrasted with the situation in quantum-dot molecules, whose interaction matrix element is governed by the overlap of just a single pair of states. While one often views the continuum as a source of decoherence, our work therefore instead suggests the possibility of using this medium to support the interaction of quantum states, a result that may allow new approaches to coherently couple nanostructures in extended geometries.

The remainder of this paper is organized as follows. Since our experimental demonstration of the continuummediated coupling is based on exploiting the specific properties of quantum point contacts (QPCs) near pinch-off, in Sec. II we first briefly review our recent work [22-26] in which we have shown how these structures may be used as an on-demand source of a localized quantum state. In Sec. III, we demonstrate the use of these devices to realize the remote-coupling scheme, showing that it gives rise to a surprisingly robust interaction. In Sec. IV, we introduce a theoretical model that reproduces the key aspects of our experiment by explicitly treating how the coupling is mediated through the continuum states. We conclude with a discussion of the implications of our findings in Sec. V.

\section{BOUND-STATE FORMATION IN QUANTUM POINT CONTACTS}

The background to our work is provided by the extensive interest that continues to focus on the nature of the electronic structure of QPCs near pinch-off, and the connection of this structure to electron transport (for recent reviews, see Refs. [27,28]). Since the carrier density at the QPC center becomes vanishingly small near pinch-off, it has been suggested that many-body phenomena can modify the electronic structure in this regime. A scenario proposed in several of these studies is that such modifications can lead to the formation of a quantum-dot-like bound state (BS), capable of localizing a single electron, at the QPC center [29-34]. The microscopic structure of this BS remains the subject of debate $[35,36]$, however, so that further experiments are called for to confirm its existence. Utilizing the FR as a key signature of the presence of a discrete state, we have implemented a mesoscopic version of the FR experiment, in which the mutual interaction between a pair of coupled QPCs reveals the signature of the BS [22-26]. In these experiments, one QPC (the detector) is configured with fixed gate bias, while the gate voltage applied to the second (swept) QPC is varied continuously, driving it to pinch-off where BS formation is expected. As illustrated schematically in Fig. 1, the two QPCs are coupled to each other through an intervening region of two-dimensional electron gas (2DEG), which provides the main means for their interaction [25]. The key observation in such experiments is that, as the swept QPC is pinched-off, an isolated resonance occurs in the detector conductance. This resonance is not a transient feature, observed only while sweeping the different gate voltages, but rather is a static signature in the detector conductance that is reproduced with high fidelity when sweeping the swept QPC either toward, or away from, pinch-off. (See the Supplemental Material [37] for further details.) Confirming that this feature is associated uniquely with physics that arises near pinch-off, no other resonances are observed as higher subbands of the swept QPC are subsequently populated. The resonance is reproduced, however, in devices with different gate configurations [22,24], in various QPCs fabricated on the same chip [24-26], and in multiple cooling cycles performed over a period of several years. From these collective observations, we are able to infer that the resonance does indeed result from the intrinsic properties of the QPC and is not a random-impurity effect.

In Ref. [23], we developed a phenomenological theory to account for the findings of our experiment, by making the initial assumption that a BS is present in the swept QPC near pinch-off. With the coupling of this BS to the detector mediated through the intervening 2DEG, it was shown that a resonance should occur in the detector conductance as the swept-QPC gate voltage drives its BS upwards through the

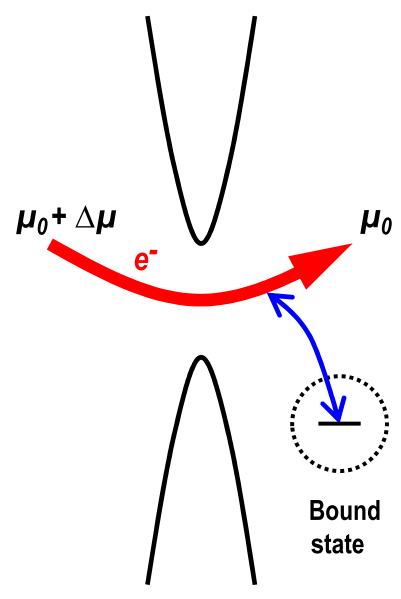

FIG. 1. Schematic illustration of the Fano resonance exhibited by a detector QPC that is coupled through a region of two-dimensional electron gas to a bound state in another QPC [22-26]. An electrochemical-potential difference between source and drain is used to drive electrons (thick red line) through the detector. A correlation then arises between the bound state and the detector, due to tunneling of electrons (thin blue line) to and from the bound state, and is resonantly enhanced when the bound-state energy matches the Fermi level in the reservoir. 
Fermi level ( $\mu_{0}$ in Fig. 1). According to this model, the resonance arises from a modification of the detector conductance due to a tunnel correlation, which develops when electrons, emerging from the detector (thick line in Fig. 1), tunnel onto the BS and back out again (thin blue line in Fig. 1). This mechanism, therefore, has all the ingredients of a Fano resonance; the transmission (and so conductance) of the detector is determined by the interference of two distinct processes, the first consisting of direct injection into the 2DEG, while the second involves initial tunneling from the detector to the BS, followed by tunneling back to the 2DEG. This Fano character was demonstrated convincingly in Ref. [25], in which we investigated the dependence of the resonance line shape (through the so-called asymmetry parameter, $q[1])$ on the separation of the two QPCs. Our experiments revealed a systematic evolution of the line shape, indicating that excitation of the two pathways responsible for the FR can be regulated directly, simply by changing the spatial arrangement of the coupled QPCs.

Taking advantage of the fact that the FR provides a highly characteristic signature of the presence of a discrete state, we have utilized the detector resonance to reveal a number of key microscopic properties of the selfconsistently formed BS on QPCs. A vital feature of these experiments is that they provide information on the microscopic state of the QPC beyond pinch-off, a regime in which direct measurements of QPC conductance yield little or no information. From studies of the temperature dependence of the detector resonance [24], we have been able to infer a robust confinement of the $\mathrm{BS}$, of $\mathrm{meV}$ order, while an in-plane magnetic field has been shown to induce Zeeman splitting into spin-up and spin-down branches [24]. From studies in which a DC bias was used to investigate the nonlinear characteristics of the detector resonance, we were able to infer the BS lever arm (its effective energy shift for a change in swept-QPC voltage) and to therefore confirm that the above-mentioned Zeeman splitting is characterized by a significantly enhanced $g$ factor $(g \sim 2)$ [26]. In the discussion that follows, we will make direct use of many of these findings to assist our analysis.

\section{EXPERIMENTAL RESULTS}

\section{A. Experimental methods}

The device that we study is that of Refs. [24-26]. It features a multigate geometry that allows coupled QPCs to be configured in various configurations [see Fig. 2(b)]. This device was realized in a high-mobility GaAs/AlGaAs quantum well (Sandia sample EA750), whose 2DEG had these features: density $2.3 \times 10^{11} \mathrm{~cm}^{-2}$, mobility $4 \times 10^{6} \mathrm{~cm}^{2} / V_{s}$, Fermi wavelength $53 \mathrm{~nm}$, and mean free path $31 \mu \mathrm{m}$ (all at $4.2 \mathrm{~K}$ ). The mean free path decreased to $4 \mu \mathrm{m}$ at $77 \mathrm{~K}$, still much longer than the largest inter-QPC separation in the device $(<1 \mu \mathrm{m})$. Using eight contacts positioned around the perimeter of the device, we

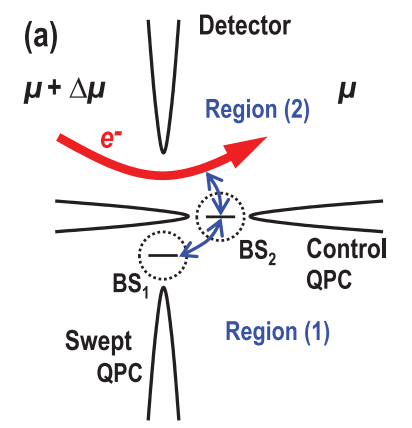

(b)

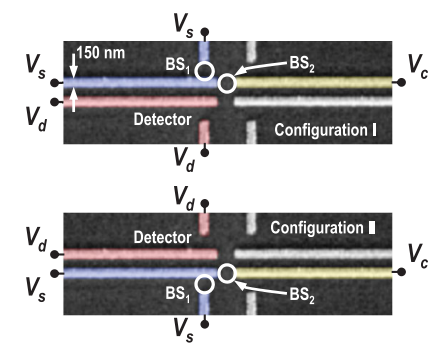

FIG. 2. (a) Schematic illustration of the manner in which we use a multi-QPC device to implement remotely interacting BSs. $\mathrm{BS}_{1}$ and $\mathrm{BS}_{2}$ are formed in the swept and control QPCs, respectively, and are coupled to each other, and to the detector, via intervening regions of $2 \mathrm{DEG}$ (thin blue lines). (b) Scanning electron micrographs of our multi-QPC device are used to illustrate the manner in which we implemented the system in (a) experimentally. Uncolored gates were held at ground potential in the experiment and so had no influence on the underlying $2 \mathrm{DEG}$.

could independently determine the conductance of the various QPCs [24-26]. Measurements (from 4.2-40 K) were performed by lock-in detection with $30 \mu \mathrm{V}$ excitation, leaving unused gates grounded to ensure that they exerted minimal influence on the resulting behavior.

\section{B. Configuring the system}

In this report, we demonstrate how the technique that we have developed for probing QPC BSs can be extended to realize systems in which two BSs, formed on separate QPCs, interact through a common 2DEG. The manner in which this is achieved is illustrated in Fig. 2, in which Fig. 2(a) is a schematic that shows the key mechanisms for interaction in the multi-QPC system. In Fig. 2(b), we use micrographs of the actual device to show the two different gate configurations that were used to implement the interacting BSs. (See the Supplemental Material [37] for a detailed description of the measurement procedure.) In both of these configurations, which are actually geometrically equivalent, the detector gates (biased at $V_{d}$ ) are indicated with red shading, while those of the swept QPC (biased at $V_{s}$ ) that defines one of the BSs $\left(\mathrm{BS}_{1}\right)$ are shown in blue. The other $\mathrm{BS}\left(\mathrm{BS}_{2}\right)$ is realized within the control QPC, which is formed by one of the gates of the swept QPC, and by the additional control gate (biased at $V_{c}$ ) highlighted in yellow. The resulting system is one in which the two BSs, being separated by a distance of as much as $400 \mathrm{~nm}$, are not coupled directly to each other, but rather they interact through a mutual 2DEG that also supports their wave-function overlap with the detector. For the purpose of the discussion below, it is important to recognize that the swept QPC $\left(\mathrm{BS}_{1}\right)$ is formed by $V_{s}$ alone, while the control QPC $\left(\mathrm{BS}_{2}\right)$ is defined by both $V_{s}$ and $V_{c}$. Consequently, as $V_{c}$ is made more negative, pinch-off of 
the control QPC should shift to the less-negative $V_{s}$, while that of the swept QPC should be unaffected.

\section{Demonstrating the continuum-mediated interaction}

Experiments were performed by measuring the variation of the detector conductance $\left(G_{d}\right)$, after first fixing $V_{c}$ and then sweeping $V_{s}$ over some fixed range. In the various panels of Fig. 3, we show the resulting $G_{d}\left(V_{s}\right)$ for several different values of $V_{c}$. Figure 3(a) corresponds to the case of least-negative $V_{c}$, for which, as $V_{s}$ is varied, the swept QPC (whose conductance is indicated by the black line) pinches-off well before the control QPC. Figure 3(f), in contrast, was obtained for the most-negative $V_{c}$, for which the control QPC (conductance shown by the green line) pinches-off first. Also plotted (as the red line) in each panel of Fig. 3 is the associated variation of $G_{d}$, which in Figs. 3(a) and 3(f) exhibits a single resonance (denoted as $R_{1}$ and $R_{2}$, respectively). As we have discussed elsewhere [22-26], the resonance serves as a detector of BS

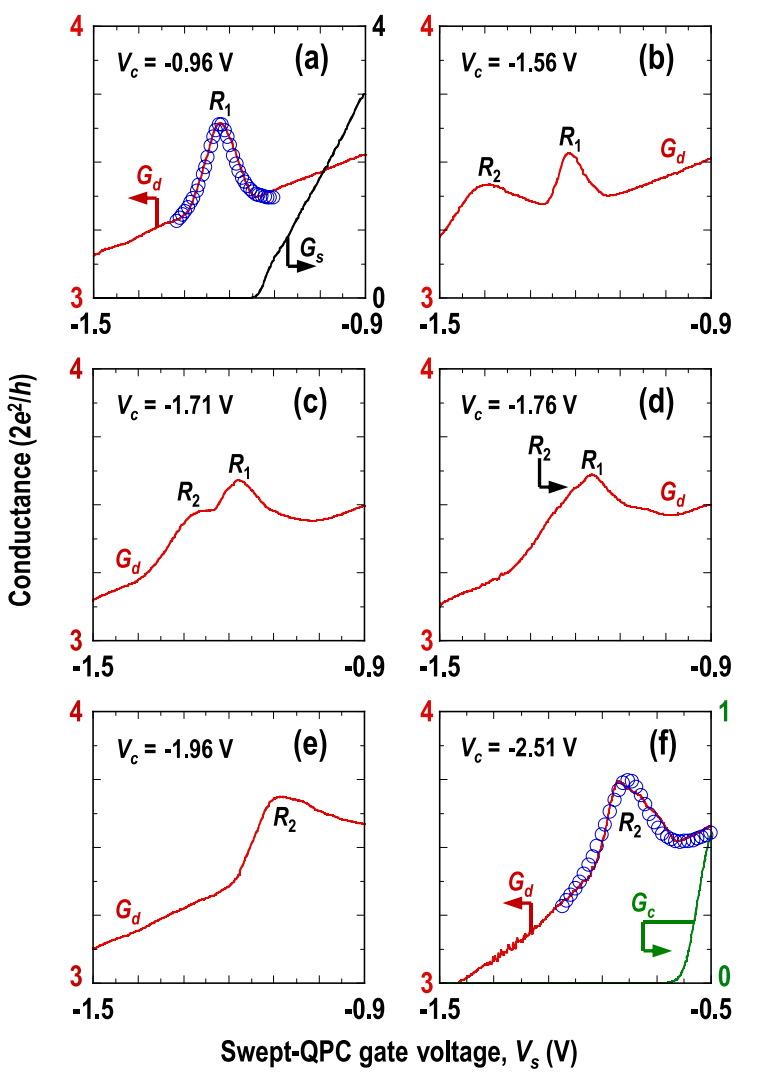

FIG. 3. Measurements of the detector conductance $\left[G_{d}\left(V_{s}\right)\right]$ for Configuration I of Fig. 2(b), at $4.2 \mathrm{~K}$ and for several different values of the control-QPC gate voltage $\left(V_{c}\right.$, indicated in each panel). Also shown in the top-left (bottom-right) panel, as the black (green) line, is the corresponding variation of the sweptQPC (control-QPC) conductance $\left(G_{s, c}\right)$, along with fits [25] (open circles) to the FR formula of Eq. (1). Top-left panel: $q=-22$ and $\Gamma=0.082 \mathrm{~V}$. Bottom-right panel: $q=-7$ and $\Gamma=0.225 \mathrm{~V}$. formation, and the correlation of $R_{1}$ and $R_{2}$ to pinch-off of the swept and control QPCs allows us to relate these resonances to $\mathrm{BS}_{1}$ and $\mathrm{BS}_{2}$, respectively. Also shown in the figures are fits of the resonances to the Fano form [1]

$$
G_{d} \propto \frac{(\varepsilon+q)^{2}}{1+\varepsilon^{2}}, \quad \varepsilon=\frac{V_{s}-V_{0}}{\Gamma / 2},
$$

where $q$ is the asymmetry parameter and $\epsilon$ and $\Gamma$ are the position and width of the resonance (measured in terms of $V_{s}$ ), respectively. (Such fitting is possible because, for a sufficiently narrow range centered around $V_{0}$, variation of $V_{s}$ translates directly to a linear shift of the BS energy, as demonstrated experimentally in Ref. [26].) According to Eq. (1), the asymmetry parameter determines the detailed line shape of the FR and physically provides information on the relative contributions of the continuum and the discrete state to the resonance [1]. A symmetric resonance is obtained when $q \rightarrow \infty$, corresponding to the case where the BS dominates, but the asymmetry grows as the role of the continuum increases. Maximal asymmetry that is a FR with a local maximum and minimum in close proximity to each other is obtained for $q=1$, which corresponds to the situation in which the discrete and continuum channels are excited with similar amplitude. In Ref. [25], we found that the asymmetry of the detector FR grows systematically as the separation of the detector and swept QPCs is reduced, consistent with an associated, and similarly systematic, evolution of the asymmetry parameter. The resonances shown in Figs. 3(a) and 3(f) are clearly well described by the form of Eq. (1), and the resulting values of the asymmetry parameter ( $q=-22$ and -7 , respectively) are in good agreement with those reported for equivalent configurations in Ref. [25].

Having discussed the details of Figs. 3(a) and 3(f), we now focus on the behavior observed in the remaining panels of Fig. 3. While Fig. 3(a) shows just a single resonance $\left(R_{1}\right)$, since the control QPC pinches-off outside the range of the figure, in Fig. 3(b) the more-negative value of $V_{c}$ causes this QPC's resonance $\left(R_{2}\right)$ to clearly appear in the data window. As $V_{c}$ is made more negative yet, there is a clear trend for $R_{2}$ to track from left to right, as pinch-off of the control QPC occurs progressively earlier. In Figs. 3(c) and 3(d), the two resonances can still be distinguished but appear very close to overlapping with each other. Finally, in Figs. 3(e) and 3(f), for which the control QPC pinches-off before the swept QPC, only a single resonance is observed. On the basis of the correlation of this resonance to the pinch-off of the control QPC [Fig. 3(f)] we identify it as $R_{2}$, an assignment that is further confirmed by the value of the asymmetry parameter characterizing this FR [25].

In Fig. 4, we present the results of measuring $G_{d}\left(V_{s}\right)$ for the two different configurations of Fig. 2(b), as we vary $V_{c}$ in a more systematic manner than in Fig. 3. The behavior exhibited in both panels of Fig. 4 is clearly very similar in 


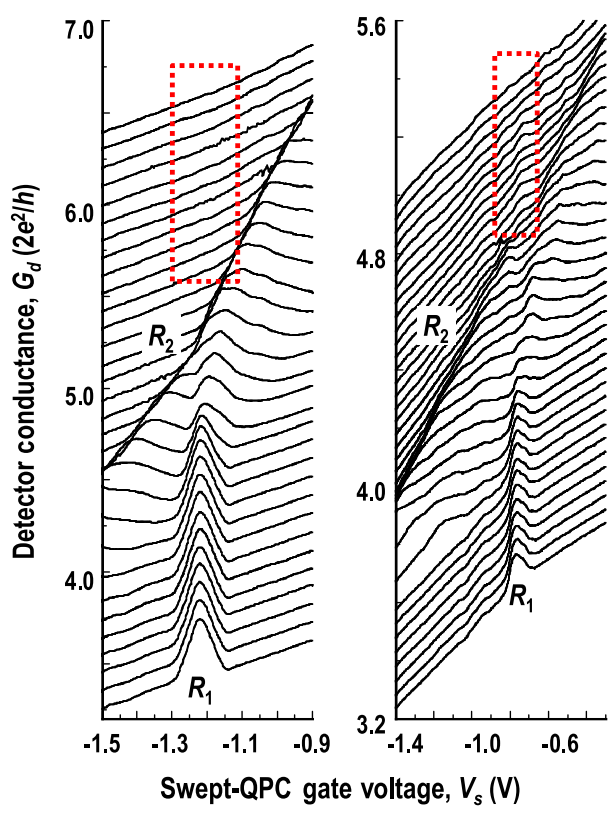

FIG. 4. The left panel shows the variation of $G_{d}\left(V_{s}\right)$ at $4.2 \mathrm{~K}$, for Configuration I of Fig. 2(b). From the lowest curve up, $V_{c}$ is incremented from $-0.96 \mathrm{~V}$ to $-2.21 \mathrm{~V}$ in $50-\mathrm{mV}$ steps, and from $-2.31 \mathrm{~V}$ to $-2.91 \mathrm{~V}$ in $100-\mathrm{mV}$ steps. Curves are shifted up in increments of $0.1 \times 2 e^{2} / h$. The right panel shows the variation of $G_{d}\left(V_{s}\right)$ at $4.2 \mathrm{~K}$, for Configuration II of Fig. 2(b). From the lowest curve up, $V_{c}$ is incremented from $-0.50 \mathrm{~V}$ to $-2.30 \mathrm{~V}$ in 50-mV steps. Curves are shifted up in increments of $0.05 \times 2 e^{2} / h$. The red dotted lines in both panels denote the missing portions of the resonance spectrum.

character, in spite of the fact that the two configurations use different gates to implement the various QPCs. That being so, the measurements of Fig. 4 represent results for two mesoscopically distinct implementations of the coupledBS system. Indeed, the two panels of this figure were obtained during different thermal cycles, performed more than a year apart, and thus truly provide measurements of microscopically distinct potential landscapes. Furthermore, we have also configured the two-BS system using other gate combinations than those shown in Fig. 2(b), and again we find behavior similar to that in Figs. 3 and 4. These various experiments therefore give us a high degree of confidence in the generic character of the behavior revealed in these figures.

With regard to the evolution of the resonances revealed in Fig. 4, this is consistent with the evolution already discussed for Fig. 3. Notably, the different dispersions of $R_{1}$ and $R_{2}$ in the $\left(V_{s}, V_{c}\right)$ plane are clearly apparent, and consistent with the manner in which the two BSs are defined; the value of $V_{s}$ at which $R_{1}$ occurs is largely insensitive to variation of $V_{c}$, while $R_{2}$ shifts to lessnegative $V_{s}$ as $V_{c}$ is made more negative. Because of these different dispersions, it might be expected that there should actually be a specific combination of $V_{s}$ and $V_{c}$ for which the two resonances should cross each other. Both panels of
Fig. 4 show an avoided crossing, however, indicating that the two peaks experience level repulsion that maintains at least a minimal separation between them [as in Fig. 3(d)]. The form of this anticrossing is quite unusual, since one of its four branches appears to be missing (as we indicate for the regions enclosed by red dotted lines). Nonetheless, it is clear for both configurations of Fig. 4 that $R_{1}$ is repelled toward less-negative $V_{s}$ as $R_{2}$ approaches it. In fact, observation of the three-branched anticrossing is a critical finding, since it confirms that the coupling among the different components of our system is mediated through the interconnecting 2DEG, rather than arising from direct electrostatic crosstalk. We arrive at this conclusion by correlating the variation of $G_{d}$ in Fig. 4 to our associated measurements of the swept- and control-QPC conductance [as in Figs. 3(a) and 3(f), for example]. In this way, we infer that the missing branch of the avoided crossing, which corresponds to the range over which the resonance $R_{1}$ should be observed, is associated with the situation for which the control QPC pinches-off before the swept QPC as $V_{s}$ is varied. Under such conditions, the wave function coupling between $\mathrm{BS}_{1}$ and the detector is suppressed, and it is for this reason that $R_{1}$ is therefore not observed. (We return to discuss this point again below.) In contrast, if $R_{1}$ were somehow associated with the charge-sensing scheme that has been used in studies of quantum dots [38], there would be no reason to expect that it should be so dramatically modified by pinching-off the control QPC.

The missing branch of the anticrossing noted above can actually be reconstructed in experiment by using an alternative measurement configuration in which the pinched-off control QPC now serves as a detector to monitor the swept QPC. This is easily achieved in experiment by making use of appropriate Ohmic contacts to the 2DEG, and results from such a configuration are shown in Fig. 5(a). This shows that the conductance $\left(G_{c}\right)$ of the control QPC exhibits a small peak that is closely correlated to the pinch-off of the swept QPC [shown in Fig. 5(b)]. (Accompanying the peak is a steplike change in $G_{c}$, which we observe quite commonly in experiments in which the detector itself is very close to pinch-off. The step most likely reflects a change in the local electrostatic environment of the control QPC, which occurs once the swept QPC fully pinches-off [38].) By utilizing the peak in Fig. 5(a) as a detector of the resonance due to $\mathrm{BS}_{1}$, in Fig. 5(c) we plot as black symbols the position of this resonance. It is clear from this figure, which also plots the resonance positions determined from the corresponding panel of Fig. 4, that the two different sets of data in Fig. 5 connect smoothly to each other. Most notably, the combination of the two data sets allows the full structure of the anticrossing between $R_{1}$ and $R_{2}$ to become clear. (It should be noted that the input impedance of the Signal Recovery 5210 lock-in amplifiers that we use is $100 \mathrm{M} \Omega$. In most of our experiments, this is many orders 

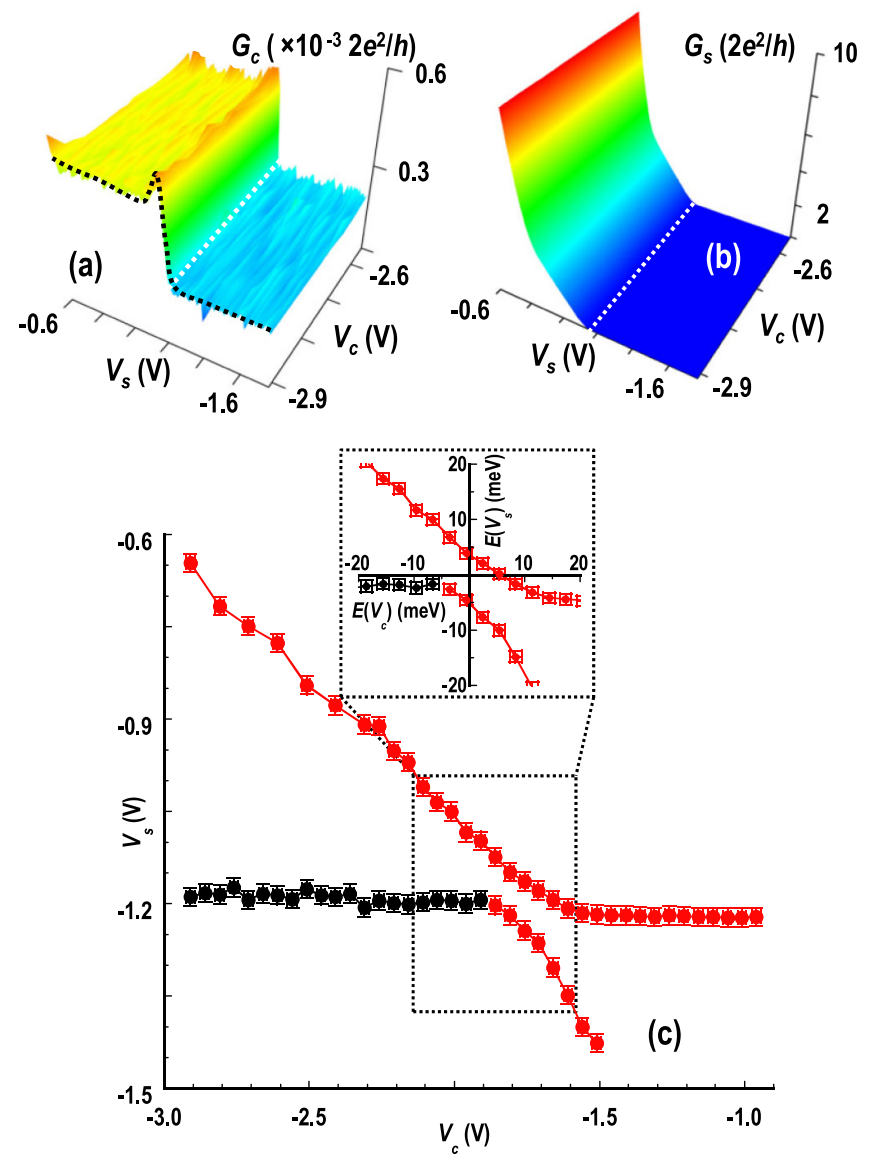

FIG. 5. (a) $G_{c}\left(V_{s}, V_{c}\right)$ for Configuration I of Fig. 2(b) at $4.2 \mathrm{~K}$. Data are for the range of $V_{s, c}$ where the control QPC is extremely close to pinch-off. (b) $G_{s}\left(V_{s}, V_{c}\right)$ for Configuration I for the same gate-voltage range and temperature as in (a). White dotted lines in (a) and (b) denote the same variation of $V_{s}-V_{c}$. The black dotted line at the front of the contour in (a) is a guide to show the line shape of $G_{c}\left(V_{s}\right)$. (c) Evolution of $R_{1}$ and $R_{2}$ for Configuration I as a function of $V_{c}$ and $V_{s}$. Black symbols were obtained using the control QPC as a detector; red symbols were inferred directly from the data of Fig. 4. The plot shown in the inset was obtained by using the lever arm [26] of the two BSs to convert variations of $V_{c, s}$ to corresponding BS-energy shifts.

of magnitude larger than the detector resistance, which is typically less than $10 \mathrm{k} \Omega$. In the measurements of Fig. 5(a), however, the measured resistance is likely loaded somewhat by the input impedance-particularly on the low-conductance side of the step after the swept QPC pinches-off-but this loading will not affect the gatevoltage position of the detector resonance, which is the main feature of interest for evaluating the avoided crossing.)

While Fig. 5(c) plots the position of the two resonances as a function of $V_{s}$ and $V_{c}$, these data can be related to equivalent energy shifts by utilizing the results of our prior, nonlinear bias-spectroscopy experiments [26]. In these, a DC bias was applied across the detector to induce a shift in the gate voltage $\left(V_{s}\right)$ position of its resonance and to therefore deduce the lever arm that connects a change of $V_{s}$ to an equivalent shift of the BS energy. Using such calibrations for the separate QPCs, in the upper inset of Fig. 5(c) we replot the data from the main panel in terms of associated energy shifts. Our plot is restricted to the region near the avoided crossing, where the level repulsion of the two resonances is clearly seen to be of order a few (2-3) meV. While such strong coupling, much larger than that $(\leq 100 \mu \mathrm{eV})$ typically reported for quantum-dot molecules [21], may seem surprising, particularly given that the two BSs are coupled only indirectly through a 2DEG, we nonetheless emphasize that our estimates are consistent with the fact that we are able to clearly observe the avoided crossing at $4.2 \mathrm{~K}$. Indeed, shortly below, we develop a theoretical model that explains the robust coupling between the two BSs precisely in terms of the fact that it is mediated by a large number of degenerate states of the intervening 2DEG.

Further insight into the nature of the coupling between the two BSs is provided by the results of the temperature(T) dependent studies that we present in Fig. 6. In Fig. 6(a), we show measurements of the detector conductance at a number of different temperatures. These data were obtained for Configuration I of Fig. 2(b), but for a different thermal cycle than the measurements of Figs. 3 and 4, after first adjusting $V_{c}$ to bring $R_{1}$ and $R_{2}$ into close proximity at the lowest temperature $(4.2 \mathrm{~K})$. In Fig. 6(b), we plot the values of the swept-QPC gate voltage $\left(V_{s}\right)$ at which the two resonances occur, from which figure we identify two distinct regimes of behavior. Over the range from 10-40 K, both $R_{1}$ and $R_{2}$ shift to more-negative $V_{s}$ with increasing temperature, a trend that we have discussed previously in our investigations of the interaction between the detector and just a single BS [24]. The reason for this behavior is easily understood and follows from the fact that, with increasing temperature, the pinch-off condition for any QPC systematically shifts to more-negative gate voltage. Consistent with the idea that it provides a signature of a BS that forms near pinch-off, the detector resonance therefore also tracks to more-negative gate voltage with increase of temperature [24], just as we see at higher temperatures in Fig. 6(b). At temperatures below $10 \mathrm{~K}$, however, very different behavior is observed; while extrapolation from higher temperatures suggests that $R_{1}$ and $R_{2}$ should approach even closer as $T$ is reduced, we instead clearly observe a crossover to peak repulsion. This is indicated by the curves with open symbols in Fig. 6(a), and its onset is also denoted by the arrow in the color contour of Fig. 6(b) [a replotting of the data of Fig. 6(a)]. In the inset to Fig. 6(c), we have used the data for $R_{1}$ to infer an effective level repulsion, by first (linearly) extrapolating the high-temperature variation of the resonance position to the lowest temperatures. After subtracting this from the actual data, and using the gate-voltage lever arm $(\gamma=$ $0.14 \mathrm{meV} / \mathrm{mV}$; see Ref. [26]) to convert from gate voltage 

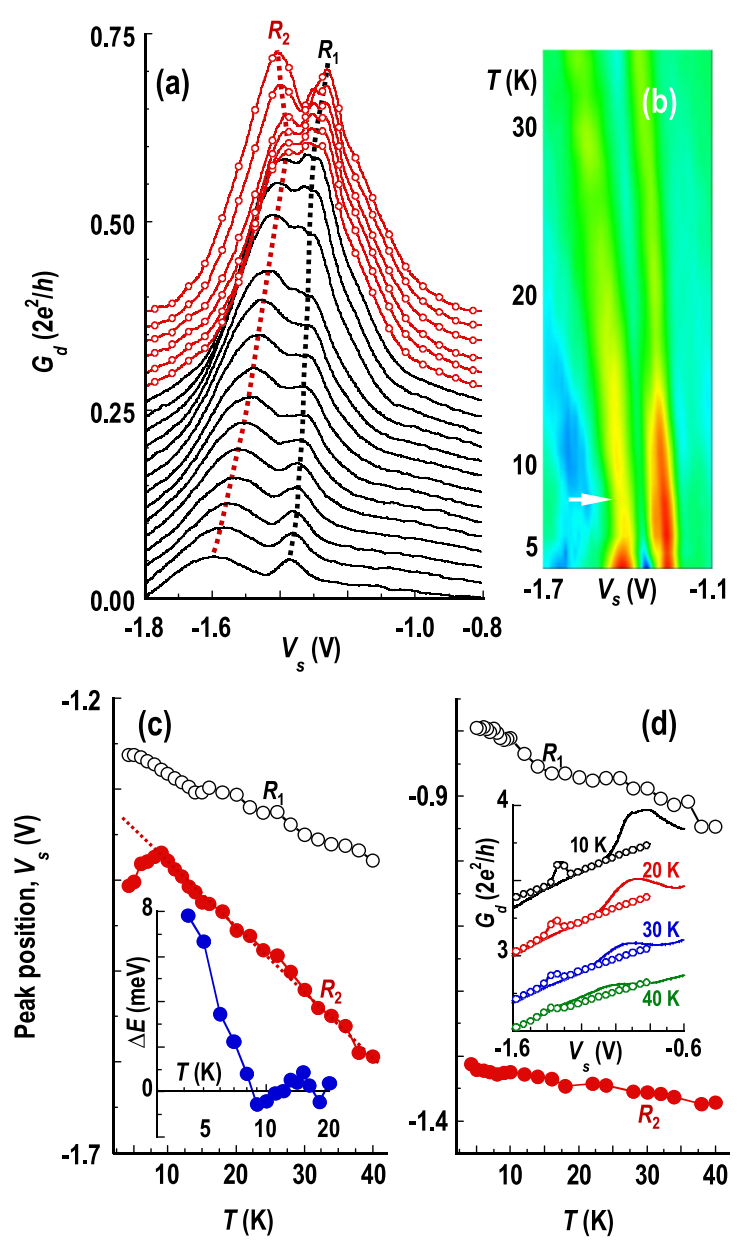

FIG. 6. (a) Measurements of the double-peak structure at different temperatures. The data are for Configuration I of Fig. 2(b) and a slowly varying background, with an average value of $3.5 \times$ $2 e^{2} / h$, has been subtracted from $G_{d}$. Successive curves are shifted upward in increments of $0.02 \times 2 e^{2} / h$. Curves with open symbols denote curves for which the peak separation increases with decreasing $T$. From bottom to top, respectively, temperatures are: 40, 36, 34, 32, 30, 28, 26, 24, 22, 20, 18, 15, 13, 11, 9, 8, 7, 6, 5, and 4.2 K. (b) Data from (a), plotted as a contour, to show appearance of peak repulsion (see arrow) below $10 \mathrm{~K}$. (c) The main panel plots the $V_{s}$ position of the two peaks $R_{1}$ and $R_{2}$ from (a) as a function of $T$. The inset plots the energy splitting inferred from the separation between the actual position of $R_{2}$ and the linear extrapolation indicated in the main figure by the dotted line. (See text for further details.) (d) The main panel plots the $V_{s}$ position of the two peaks $R_{1}$ and $R_{2}$, obtained with $V_{s}$ and $V_{c}$ configured so that the two resonances are far from their avoided crossing. The inset illustrates the temperature dependence of the two resonances under these conditions. The results of two separate measurements are combined at each temperature, since each measurement exhibits just one resonance (due to $\mathrm{BS}_{1}$ or $\mathrm{BS}_{2}$ ).

to a BS-energy shift, we thus arrive at the inset to Fig. 6(c). According to this plot, the level repulsion grows from a value of zero near $10 \mathrm{~K}$ to reach as much as $8 \mathrm{meV}$ at $4.2 \mathrm{~K}$, a value within a factor of 2 of the level repulsion inferred from the inset to Fig. 5(c).
Importantly, the peak repulsion apparent in the results of Figs. 6(a)-6(c) is found only when the two gate voltages $\left(V_{s}\right.$ and $\left.V_{c}\right)$ are in close proximity to each other. This is confirmed by the results of Fig. 6(d), which show the corresponding temperature-dependent evolution of $R_{1}$ and $R_{2}$, measured under conditions where they are detuned significantly from the anticrossing. Clearly, there is no evidence of the peak repulsion in this figure; instead, the individual resonances shift monotonically to morenegative gate voltage with increasing temperature, for the reasons that we have already discussed. It is also worth noting that the individual resonances survive to temperatures beyond $30 \mathrm{~K}$ [as in Fig. 6(a)], indicating that the effective confinement of the associated BSs should be of meV order, just as we have demonstrated previously in our work on single-BS detection [24-26].

\section{THEORETICAL ANALYSIS}

\section{A. Modeling the system}

The key result of our experiment is that a robust interaction, significantly larger than that typical of quantum-dot molecules, may be generated between a pair of remote quantum states by coupling them through a continuum. For insight into the mechanism that supports this interaction, we model the experiment as indicated in Fig. 2(a). Our description of the system in this manner is motivated by our understanding of the way in which the selfconsistent potential of a QPC should evolve in a scenario involving BS formation. Prior to pinch-off, while the QPC is conducting, its potential consists of a two-dimensional saddle that supports a set of equally spaced harmonicoscillator levels [21]. As the gate voltage is made more negative, the lateral confinement of electrons inside the QPC increases, and these one-dimensional subbands are driven upward in energy. As pinch-off is approached, the many-body interactions that arise from the locally reduced electron density around the QPC are thought to fundamentally alter the saddle potential, generating a local minimum at its center that supports the BS. Although the precise manner in which the BS evolves with gate voltage is not completely understood, its confinement presumably increases as the QPC is driven from just below the last plateau toward full pinch-off (where, as the QPC barrier is eventually driven far above the Fermi level, the BS must presumably vanish.) Over this range, the QPC may essentially be considered as a naturally formed quantum dot, whose nanoscale dimensions should result in a significant charging energy (denoted here as $U_{n}$ for $\mathrm{BS}_{n}, n=1,2$ ) for the addition of a second electron $[23,29]$. Indeed, our prior experiments [24] suggest that this charging energy is of meV order, consistent with numerical estimates [29]. With these considerations, the local density of states of a QPC may be represented as a single discrete state, which lies below a set of transverse oscillator levels with free dispersion in the direction of current flow. When the QPC is open, 
its BS should lie well below the Fermi level so that the regular one-dimensional subbands govern the conductance. As pinch-off is approached, however, and the entire level structure is pushed upward, the discrete state nears the Fermi level and is then able to influence transport. With the above picture in mind, we can return to Fig. 2(a), which shows that we describe our experiment in terms of two localized states, $\mathrm{BS}_{1}$ in the swept $\mathrm{QPC}$ and $\mathrm{BS}_{2}$ in the control QPC, that are coupled to a detector, and to each other, via 2DEG in Regions (1) and (2). Our objective is to calculate the manner in which the detector conductance then reflects the interaction between the BSs, and we achieve this by starting from the Hamiltonian (whose formulation in the language of second quantization is presented in the Supplemental Material [37])

$$
\begin{aligned}
\mathcal{H}= & \mathcal{H}_{\mathrm{det}}+\mathcal{H}_{\mathrm{con}}+\mathcal{H}_{\mathrm{swe}}+\mathcal{H}_{(1)}+\mathcal{H}_{(2)} \\
& +\mathcal{H}_{\mathrm{BS}_{1}}+\mathcal{H}_{\mathrm{BS}_{2}}+\mathcal{H}_{\mathrm{int}} .
\end{aligned}
$$

The first three terms on the right-hand side of this equation represent, respectively, the states of the detector, the control QPC and the swept QPC. These states correspond (see the Supplemental Material [37]) to noninteracting one-dimensional subbands that are hybridized with freeelectron-like states in 2DEG regions (1) and (2). The fourth and fifth terms in Eq. (2) describe the 2DEG states in these regions, while the sixth and seventh terms represent the two BSs:

$$
\mathcal{H}_{\mathrm{BS}_{n}}=\sum_{\sigma} \varepsilon_{n \sigma} n_{n \sigma}+U_{n} n_{n \uparrow} n_{n \downarrow},
$$

where $\epsilon_{n \sigma}$ is the energy of $\mathrm{BS}_{n}(n=1,2)$ for spin projection $\sigma, U_{n}$ is the charging energy noted already, and $n_{n \sigma}$ is the charge-number operator. [There is no term in Eq. (2) for a BS in the detector, since this typically has a conductance larger than $2 e^{2} / h$ and its BS is therefore either not formed or exerts no influence on transport.] While the two BSs are taken to interact with electrons in the 2DEGs via tunneling, for simplicity we neglect any interaction between each BS and the one-dimensional subbands in its QPC, since such an interaction should not fundamentally affect our main conclusions.

The last term in Eq. (2) describes the interaction between the different components of the system, where, to ensure a close connection to experiment, we take $\mathrm{BS}_{1}$ to be coupled to Region (1) while $\mathrm{BS}_{2}$ is coupled to Regions (1) and (2). It is this common coupling to the same region of 2DEG that provides an effective interaction between the BSs, as can be shown explicitly by considering the subsystem comprising the two BSs and the 2DEG of Region (1). Through application of the Schrieffer-Wolff transformation [39], we show in the Supplemental Material [37] that the Hamiltonian for this subsystem can be recast as

$$
\mathcal{H}_{\mathrm{BS}}=-\frac{1}{2} \sum_{n m \sigma} W_{n m} d_{n \sigma}^{\dagger} d_{m \sigma}+\text { charging interactions. }
$$

This form implies a direct interaction between the BSs that can be expressed as $W_{12} d_{1 \sigma}^{\dagger} d_{2 \sigma}$, where the operators $d_{n \sigma}^{\dagger}$ $\left(d_{n \sigma}\right)$ create (annihilate) electrons at $\mathrm{BS}_{n}$. In the Supplemental Material [37], we show that the interaction potential is given by

$$
W_{n m}=\sum_{\mathbf{k}} v_{\mathbf{k} \sigma n}^{*} v_{\mathbf{k} \sigma m}\left(\frac{1}{E_{\mathbf{k}}-\varepsilon_{n \sigma}}+\frac{1}{E_{\mathbf{k}}-\varepsilon_{m \sigma}}\right),
$$

where, crucially, the summation runs over all electron states of the 2DEG (both occupied and unoccupied states with momentum $\mathbf{k}$ and energy $E_{\mathbf{k}}$ ) and the coupling of these states to $\mathrm{BS}_{n}$ is described by the matrix element $v_{\mathbf{k} \sigma n}$. As the two BSs approach each other in energy $\left(\varepsilon_{1 \sigma}=\varepsilon_{2 \sigma}\right.$, which we achieve in the experiment by suitable tuning of $V_{s}$ and $V_{c}$ ) and for $E_{\mathbf{k}}$ close to the Fermi energy ( $E_{F}$, which is taken here as the zero of energy)

$$
W_{n m}\left(\varepsilon_{\mathbf{k} \boldsymbol{\sigma}}=E_{F}\right) \sim-2 \frac{\left|v_{n}\right|^{2}}{\varepsilon_{n \sigma}},
$$

where $v_{n}=v_{\mathbf{k} \sigma n}=v_{\mathbf{k} \sigma m}$. The key result that follows from this form is that the coupling $W_{n m}$ diverges as the BS energies approach the Fermi level in the 2DEG reservoir; under this condition, we have $\left|\varepsilon_{n \sigma} / v_{n}\right| \lesssim 1$, and the coupling $\left|W_{n m}\right| \gtrsim 2\left|v_{n}\right|$. That is, the effective coupling of the two BSs, mediated through the 2DEG, is much larger than the hybridization of either BS with the 2DEG $\left(v_{n}\right)$. Formally, this result arises from the fact that the inter-BS coupling is mediated by all (both occupied and unoccupied) states of the intervening 2DEG. The only assumption that should hold to ensure the validity of this model is that transport between the two BSs be ballistic, a requirement that is reasonable in our experiments, performed on highmobility 2DEG systems at low temperatures.

In addition to establishing the presence of a 2DEGmediated coupling between the BSs, we are also interested in understanding how this coupling is manifested in the detector conductance. For this purpose, in the Supplemental Material [37], we modify the BS subsystem of Eq. (4) by adding a coupling through Region (2) to the detector. After solving the resulting Hamiltonian with Green-function techniques, we are then able to calculate the local density of states (LDOS) for the BS subsystem and to compute the modification to the detector conductance due to the coupling to the two BSs.

\section{B. Simulation results}

The key findings of our theoretical model are presented in Fig. 7, in which we compare calculations of the LDOS and the detector conductance for the system of Fig. 2(a). While the manner in which these calculations are performed is described in detail in the Supplemental Material [37], we emphasize here that our choice of key parameters is motivated directly by feedback from experiment: (1) In Ref. [26], we showed from bias spectroscopy 
that a change of gate voltage translates to a linear shift in BS energy. In Fig. 7, we therefore represent the effect of varying $V_{c}$ or $V_{s}$ as associated BS-energy shifts, and it should be noted that the range of these axes (in meV) is similar to that in the inset to Fig. 5(c). (2) As in experiment, the energy of $\mathrm{BS}_{1}$ is taken to be determined by $V_{s}$ alone, while that of $\mathrm{BS}_{2}$ is depends on both $V_{s}$ and $V_{c}$. For convenience, we take the energy of $\mathrm{BS}_{2}\left(\epsilon_{2 \sigma}\right)$ to be zero whenever $V_{s}+V_{c}=0$ in Fig. 7. This does not affect our quantitative conclusions regarding the inter-BS interaction, however. (3) The noninteracting one-dimensional subbands of the swept and detector QPCs are taken as harmonic-oscillator levels with a spacing $\left(\omega_{p}=\omega_{q}=\right.$ $1 \mathrm{meV}$ ) consistent with bias spectroscopy of such structures [40]. For positive gate voltages $\left(V_{s}>0\right)$, the subbands in the swept QPC lie below the Fermi level, while negative $V_{s}$ corresponds to the regime where the swept QPC is pinched-off. In this situation, only $\mathrm{BS}_{1}$ is occupied in this QPC. For the detector QPC, the subband bottoms are taken as $-20+m \omega_{p}$, where $\omega_{p}=1 \mathrm{meV}$ and $m=$ $1, \ldots, 20$. The detector is therefore in the multimode limit, although this does not significantly affect our conclusions. (4) The charging energies that appear in Eq. (3) are taken as $U_{n}=1.5 \mathrm{meV}$, a choice that is motivated by the results of self-consistent calculations of the QPC electronic structure [29] and by our own experiment [24]. (5) Temperature is included through its influence on the different number operators, via the Fermi distribution, and is taken here to be $4.2 \mathrm{~K}$ to match experiment.

In Figs. 7(d) and 7(f), we show the corresponding contributions of the two BSs to the detector conductance,

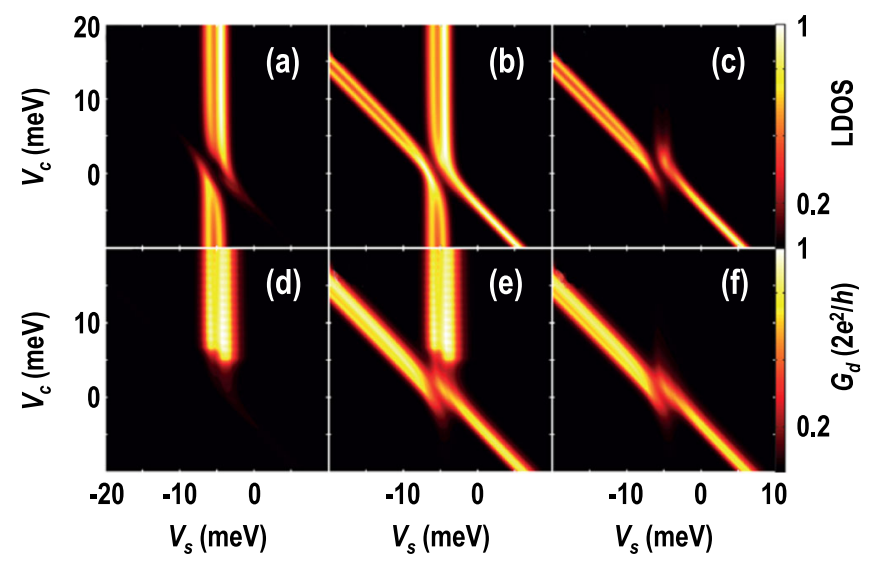

FIG. 7. The upper row of panels shows the calculated LDOS for (a) $\mathrm{BS}_{1}$, (b) $\mathrm{BS}_{1}$ and $\mathrm{BS}_{2}$, and (c) $\mathrm{BS}_{2}$ as functions of the gate voltages $V_{s}$ and $V_{c}$ (expressed as BS-energy shifts, in meV). As in the experiment, $\mathrm{BS}_{1}$ is defined by $V_{s}$ alone while $\mathrm{BS}_{2}$ is formed by $V_{s}$ and $V_{c}$. The lower row of panels shows the correction to the detector conductance, calculated from Eq. 35 in the Supplemental Material [37]. (d) Contribution from first term. (e) Contribution from both terms. (f) Contribution from the second term. A description of the parameter values used in these calculations is provided in the Supplemental Material [37]. obtained by numerical calculation of Eq. 35 in the Supplemental Material [37]. Figure 7(d) shows the variation of the second term on the right-hand side of this equation and represents the correction to the detector conductance due to its coupling to $\mathrm{BS}_{1}$. Figure 7(f) similarly shows the correction to the conductance due to its coupling to $\mathrm{BS}_{2}$, provided by the first term of Eq. 35. Figure 7(e) represents the full variation of the detector conductance, obtained by summing both of these terms, and so it is the quantity that should be compared with the results of experiment. In Fig. 7(e), we see a resonance in the detector conductance due to $\mathrm{BS}_{2}$, which precisely follows the evolution of this state [shown in Fig. 7(c)]. In marked contrast, Fig. 7(d) plots the contribution to $G_{d}$ from $\mathrm{BS}_{1}$, but it shows only the resonance due to this BS for $V_{c}>$ $5 \mathrm{meV}$, in spite of the fact that the LDOS confirms the presence of this state over the entire range of the plot. Consequently, in Fig. 7(e), where we plot the total contribution to the detector conductance due to the two BSs, rather than obtaining the full anticrossing revealed in Fig. 7(b), we instead obtain the three-branched structure that is highly reminiscent of our experiment. Consistent with our experiment, the reason for this unusual structure is readily identified: The missing branch of the avoided crossing occurs when the coupling of $\mathrm{BS}_{1}$ to the detector QPC becomes cut off, due to the full depletion of the intervening control QPC. Under these conditions, there is no wavefunction overlap between the detector and $\mathrm{BS}_{1}$, and so the detector's conductance is featureless. The very clear correspondence between our experiment and these simulations provides strong support for the notion that the interaction between the two BSs in our system is indeed mediated through the intervening continuum.

Turning now to the results of Fig. 7, Figs. 7(a) and 7(c) show the calculated LDOS (as a function of $V_{c}$ and $V_{s}$ ) for $\mathrm{BS}_{1}$ and $\mathrm{BS}_{2}$, respectively, and clearly reveal the signature of a localized state in each QPC. In both of these panels, the peak that is observed in the LDOS is actually split into two components, which are separated by the charging energy of $1.5 \mathrm{meV}$ and indicate the population of the associated BS by its first and second electrons. Consistent with the experiment, the $V_{s}$ position of $\mathrm{BS}_{1}$ is unaffected by variation of $V_{c}$, while $\mathrm{BS}_{2}$ shifts to lessnegative $V_{s}$ as $V_{c}$ is made more negative. Over the range where a crossing of the two BSs would be expected, their respective LDOS are strongly modified, and Fig. 7(b), which plots the cumulative LDOS for the two BSs, clearly reveals that this behavior is associated an avoided crossing of these BSs. Reading off a tunnel splitting from the structure of this avoided crossing, we obtain a value of around $3 \mathrm{meV}$, in good agreement with our experiment [Fig. 5(c)].

The different configurations that are achieved in the twoBS system as the gate voltages $\left(V_{s, c}\right)$ are varied are summarized in Fig. 8. In the upper part of this figure, we replot 

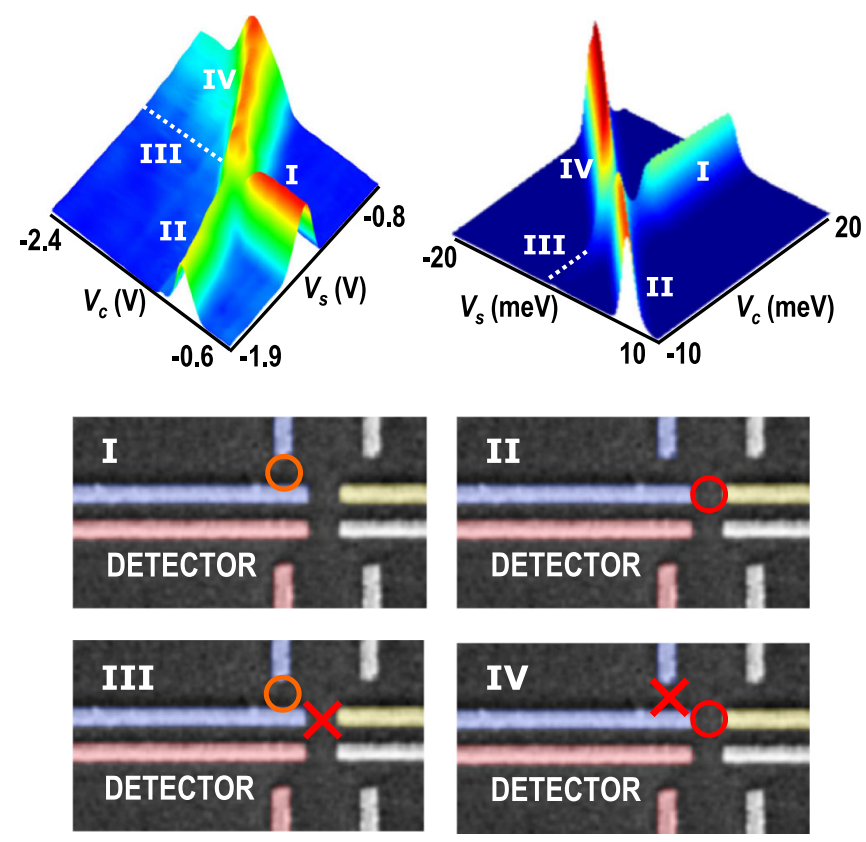

$\mathrm{BS}_{1}$ $\mathrm{BS}_{2}$

\section{Pinched-off QPC}

FIG. 8. The upper-left panel shows the data of Fig. 4 (Configuration I), replotted as a 3D contour, while the upperright panel shows a similar replotting of Fig. 7(e). The lower four panels use electron micrographs of the device to indicate its configuration, for each of the four resonances (I through IV) identified in the upper $3 \mathrm{D}$ contours.

(from Figs. 4 and 7) the measured and calculated variations of $G_{d}\left(V_{s, c}\right)$ as three-dimensional contours. This comparison is particularly useful since it demonstrates the extent to which our calculations achieve a high degree of quantitative accuracy relative to the experiment. In the lower part of the figure, we use electron micrographs of the device to denote the relationship of the different branches of the resonance spectrum (numbered I through IV in the 3D contours) to the state of the swept and control QPCs. As we indicate in Figs. 9(a) and 9(b), these different regions are related to discrete changes in the number of electrons that occupy the two BSs. Figure 9(a) shows the total population of the two-BS system (see the Supplemental Material [37] for further details) and exhibits distinct regions for which the total electron number $(N)$ associated with the BSs ranges from zero to four. [This plot should be compared with Fig. 7(b).] In Fig. 9(b), on the other hand, we show how the two separate BSs contribute to this change in electron number. We mentioned already that the LDOS exhibits a double-peak structure [see Fig. 7(b), for example], which arises from the population of each BS by its first and second electron. According to our theory, the energy separation of these two peaks is simply given by the BS on-site energy $\left(U_{n}\right)$, which is taken here to be $1.5 \mathrm{meV}$. Evidence of such a doublet structure has been found in prior experiment [24].
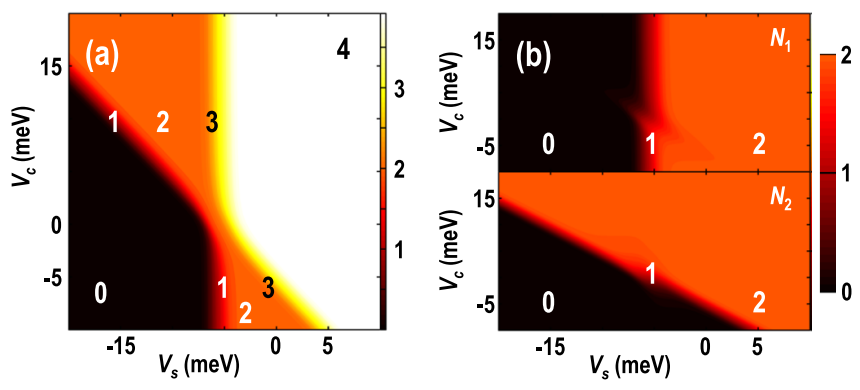

FIG. 9. (a) Total electron population of the two BSs and its variation with $V_{c, s}$. (b) Corresponding populations $\left(N_{1}\right.$ and $\left.N_{2}\right)$ of the two separate $\mathrm{BSs}\left(\mathrm{BS}_{1}\right.$ and $\mathrm{BS}_{2}$, respectively) for the same variation of $V_{c, s}$.

\section{CONCLUDING REMARKS}

Our experiment has demonstrated a robust coupling between a pair of BSs, significantly stronger than that which typically arises from the direct wave-function overlap in quantum-dot molecules, by coupling the two states through a continuum. Formally, we have explained this result within the framework of the Schrieffer-Wolff transformation by noting that the effective interaction between the BSs is essentially mediated by all states of the intervening continuum to which the BSs are separately coupled. The implication of our analysis is that we can essentially replace the setup consisting of the two BSs and their intervening 2DEG [Fig. 10(a)] with an effective model that more closely resembles the double-well potential characteristic of quantum-dot molecules [Fig. 10(b)]. In

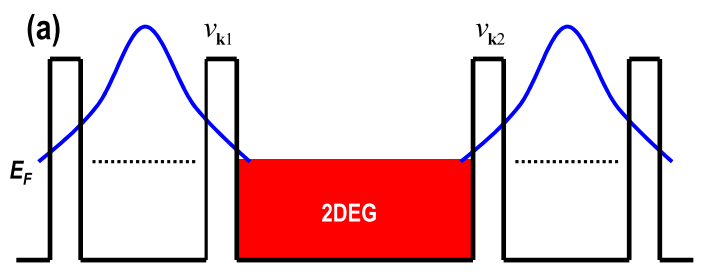

(b)

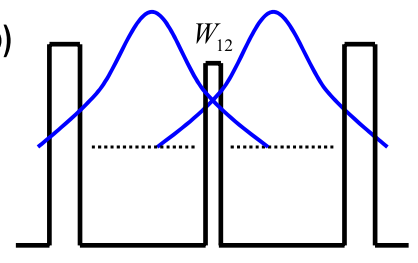

FIG. 10. (a) Schematic potential diagram showing two BSs formed on QPCs that are separated by an intervening 2DEG. The coupling of each BS to this reservoir is denoted by the matrix element $v_{\mathbf{k} \sigma 1(2)}$. (b) The system shown in (a) is replaced here with an equivalent setup consisting of two BSs that are directly coupled through a thin tunnel barrier, with an effective tunneling rate of $W_{12}$. 
this representation, the two BSs can be considered to effectively be directly coupled to each other by a potential barrier that is actually lower than the barriers that couple the BSs to the 2DEG. With this coupling strength denoted as $W_{n m}$ [as defined in Eq. (5)], the two BSs should form coherent molecular states with energies

$$
E_{ \pm \sigma}=\frac{1}{2}\left(\sum_{n}\left(\varepsilon_{n \sigma}+W_{n}\right) \pm \sqrt{\Delta^{2}+4\left|W_{12}\right|^{2}}\right)
$$

where $\Delta=\varepsilon_{1 \sigma}+W_{1}-\varepsilon_{2 \sigma}-W_{2}$ is the detuning of the BSs. At resonance we have $\Delta=0$, so that the effective tunnel splitting of the BSs reduces to just $\left|E_{+\sigma}-E_{-\sigma}\right|=$ $2\left|W_{12}\right|$. As we have noted already [see the discussion of Eq. (6)], $W_{12}$ can be much larger than $v_{\mathbf{k} \sigma 1(2)}$, and it is this characteristic that leads us to replace the system of Fig. 10(a) with that of Fig. 10(b). A comment that should be made here concerns the relative amplitude of the confining barriers that localize the BS on a QPC, and those barriers that control the coupling to the external reservoirs in conventional, Coulomb-blockaded, quantum dots. While our temperature-dependent studies [24] suggest that the effective barriers induced in QPCs should be around a few meV high, those in intentionally formed quantum dots are expected to be significantly higher ( $>10 \mathrm{meV}$, for example) and wider. This difference in barrier strength should also be important for enhancing the interdot coupling in our experiments.

In the Schrieffer-Wolff transformation that we have used to describe the interaction between the two BSs, the robust character of this interaction is understood as arising from the fact that each of the BSs is coupled to a highly degenerate set of states in the 2DEG. One aspect of our experiment that we have not addressed, however, concerns the temperature-dependent studies of Fig. 6. These reveal that the level repulsion of the two BSs appears only when the temperature is reduced below $10 \mathrm{~K}$ and that for temperatures higher than this the BSs appear independent of each other. At present, we are unable to account for this behavior within our model, in which the role of temperature is simply included through Fermi-Dirac statistics. The significance of this temperature cutoff is suggested, however, by considering the characteristic length scales that govern coherent transport in the 2DEG that couples the BSs. The first of these lengths is the dephasing length, which is the characteristic distance traveled between dephasing events and can be estimated by noting that, for 2DEG systems, a typical value for the dephasing time is around $1 \mathrm{ps}$ at $10 \mathrm{~K}$ [41]. For the Fermi velocity $\left(v_{F}=2 \times 10^{5} \mathrm{~m} / \mathrm{s}\right)$ in our high-mobility $2 \mathrm{DEG}$, this translates to a ballistic coherence length of around $200 \mathrm{~nm}$. The other length scale is the thermal length, which, for ballistic transport, is expressed as $L_{T}=\hbar v_{F} / k_{B} T$, yielding $L_{T}=160 \mathrm{~nm}$ at $10 \mathrm{~K}$ with the aforementioned $v_{F}$. From these estimates we therefore see that $10 \mathrm{~K}$ corresponds to a characteristic crossover temperature, at lower values than which the 2DEG should support coherent coupling between the BSs, while at higher temperatures it should become incoherent. One possibility, therefore, is that the enhancement of the tunnel splitting that we see below $10 \mathrm{~K}$ (Fig. 6) is connected to the temperature dependence of the coherence lengths, leading to a larger number of states contributing to $W_{n m}$ at lower temperatures. Further theoretical studies are required, however, to clarify this point, which at present is beyond the scope of our calculations.

In this work, we have focused on explaining the observed resonances in the conductance of the detector QPC and have emphasized the origin of these features in terms of a continuum-mediated coupling between the BSs. While our theoretical analysis quantitatively establishes this interpretation, we nonetheless should at least consider the possibility of other mechanisms. The first is just a capacitive coupling among the various components of the system, analogous to that which arises in charge-sensing experiments (such as Ref. [38]). In such charge sensing, however, the QPC that serves as the detector must be formed in very close proximity to the quantum dot that it monitors (in fact, it is usually integrated into this structure), and it must furthermore be configured close to pinch-off to allow its conductance to be sensitive to single-electron charging of the dot. This is very different from the situation in our experiments, in which the detector QPC is separated from the BSs that it monitors and in which its conductance is typically well above pinch-off. Under such conditions, it is reasonable to expect that the detector should be largely insensitive to electrostatic effects arising from singleelectron charging of a remote bound state. In fact, in Ref. [24] we showed that the amplitude of the detector resonance is dramatically reduced as pinch-off is approached (behavior that is also apparent in Fig. 4 here), which is actually the opposite dependence from that found in usual charge-sensing experiments. Another possible mechanism of interaction is an RKKY effect, reminiscent of reports for quantum dots [42-44]. Such a mechanism would also be mediated through the intervening 2DEG and is actually accounted for via the exchange term $\left(J_{n \sigma}(k)\right)$ that appears in Eq. 9 of the Supplemental Material [37]. Our results of Figs. 7 and 8 were obtained by neglecting the role of this exchange term, however, which suggests that an RKKY mechanism is not required to produce the effects that we observe. Indeed, in prior work on the RKKY mechanism in quantum dots, we note that the coupling was suppressed as the temperature was increased to just $1 \mathrm{~K}$ [44], an order of magnitude smaller than the temperature cutoff that we find in our experiment. At the same time, however, we cannot definitively rule out the possibility that this mechanism may also contribute to the behavior that we observe. In fact, we note the presence of some remnant minor structure in the missing branch of the avoided crossing of the right panel of Fig. 4, which may 
point to the presence of interaction beyond that which we consider. We do not have a definitive conclusion regarding this point at present, but this should not affect our main conclusions regarding the origins of the BS coupling.

An interesting aspect of our experimental results that we have not thus far emphasized is that the system we have implemented provides a mesoscopic realization of a multistate FR. While FRs have been investigated for more than half a century, by far the vast majority of these investigations have focused on the interference arising from coupling a continuum to just a single discrete state. In his original analysis, however, Fano also predicted the possibility of more-complicated multistate resonances, in which two or more discrete states interfere with each other through their coupling to a common continuum [1]. Examples of such behavior are rare, however, with the phenomenon of $q$ reversal due to intruder states in Rydberg atoms having been most widely studied [6-9]. In this effect, the discrete series of FRs arising from a specific manifold of levels in a Rydberg atom is strongly modified by its interference with other manifolds in the same energy range. That being so, this behavior is therefore a one-atom effect, in which the levels involved in the multistate resonance all arise within the same atom. This should be contrasted with the situation in our experiment, in which the component states involved in the multistate resonance are realized on spatially remote nanostructures, making this behavior a truly nonlocal phenomenon. While one often views the continuum as a source of decoherence, our work therefore suggests the possibility of using this medium to support the interaction of quantum states, a result that has the potential of opening up new approaches to coherently couple nanostructures in complex geometries. The nonlocal coupling considered here has been proposed, for example, as a means to achieve an electronic analog of the Dicke effect [16,45], a wellknown effect in quantum optics [46,47] in which superradiance arises when a photon field mediates the interaction of excited atoms. Our demonstration of extended-molecule formation demonstrates the potential of realizing new electronic devices by transferring concepts from quantum optics.

\section{ACKNOWLEDGMENTS}

This work was supported by the Department of Energy (DE-FG03-01ER45920) and was performed, in part, at the Center for Integrated Nanotechnologies, a U.S. DOE, Office of Basic Energy Sciences nanoscale science research center. Sandia National Laboratories is a multiprogram laboratory operated by Sandia Corporation, a Lockheed-Martin Company, for the U.S. Department of Energy under Contract No. DE-AC04-94AL85000. J.F. thanks the Swedish Research Council (622-2007-562) for support. L. M. is partially supported by the PSC-CUNY, Grant No. 64316-00 42.
[1] U. Fano, Effects of Configuration Interaction on Intensities and Phase Shifts, Phys. Rev. 124, 1866 (1961).

[2] U. Fano and A. R. P. Rau, Atomic Collisions and Spectra (Academic Press, New York, 1986).

[3] A. Giusti-Suzor and H. Lefebvre-Brion, Theoretical Study of Complex Resonances near Ionization Thresholds: Application to the $\mathrm{N}_{2}$ Photoionization Spectrum, Phys. Rev. A 30, 3057 (1984).

[4] C. H. Greene and C. Jungen, Molecular Applications of Quantum Defect Theory, Adv. At. Mol. Phys. 21, 51 (1985).

[5] J.P. Connerade, Highly Excited Atoms (Cambridge University Press, Cambridge, England, 1998).

[6] J. P. Connerade and A. M. Lane, Interacting Resonances in Atomic Spectroscopy, Rep. Prog. Phys. 51, 1439 (1988).

[7] D. Wintgen and H. Friedrich, Perturbed Rydberg Series of Autoionizing Resonances, Phys. Rev. A 35, 1628 (1987).

[8] Q. Wang and C.H. Greene, Parameter-Dependent Multichannel Rydberg Spectra, Phys. Rev. A 44, 1874 (1991).

[9] M. Domke, C. Xue, A. Puschmann, T. Mandel, E. Hudson, D. A. Shirley, G. Kaindl, C. H. Greene, H. R. Sadeghpour, and H. Petersen, Extensive Double-Excitation States in Atomic Helium, Phys. Rev. Lett. 66, 1306 (1991).

[10] J. Göres, D. Goldhaber-Gordon, S. Heemeyer, M. A. Kastner, H. Shtrikman, D. Mahalu, and U. Meirav, Fano Resonances in Electronic Transport through a SingleElectron Transistor, Phys. Rev. B 62, 2188 (2000).

[11] K. Kobayashi, H. Aikawa, S. Katsumoto, and Y. Iye, Tuning of the Fano Effect through a Quantum Dot in an Aharonov-Bohm Interferometer, Phys. Rev. Lett. 88, 256806 (2002).

[12] J. Kim, J.-R. Kim, J.-O. Lee, J. W. Park, H. M. So, N. Kim, K. Kang, K.-H. Yoo, and J.-J. Kim, Fano Resonance in Crossed Carbon Nanotubes, Phys. Rev. Lett. 90, 166403 (2003).

[13] A. C. Johnson, C. M. Marcus, M. P. Hanson, and A. C. Gossard, Coulomb-Modified Fano Resonance in a OneLead Quantum Dot, Phys. Rev. Lett. 93, 106803 (2004).

[14] A. M. Satanin and Y.S. Joe, Fano Interference and Resonances in Open Systems, Phys. Rev. B 71, 205417 (2005).

[15] A. Fuhrer, P. Brusheim, T. Ihn, M. Sigrist, K. Ensslin, W. Wegscheider, and M. Bichler, Fano Effect in a Ring-Dot System with Tunable Coupling, Phys. Rev. B 73, 205206 (2006).

[16] A. V. Malyshev, P. A. Orellana, and F. Dominguez-Adame, Quantum Electron Splitter Based on Two Quantum Dots Attached to Leads, Phys. Rev. B 74, 033308 (2006).

[17] M. Kroner, A. O. Govorov, S. Remi, B. Biedermann, S. Seidl, A. Badolato, P. M. Petroff, W. Zhang, R. Barbour, B.D. Gerardot, R. J. Warburton, and K. Karrai, The Nonlinear Fano Effect, Nature (London) 451, 311 (2008).

[18] Y. Berlatzky and S. Klaiman, Controlling Fano Profiles via Conical Intersections, Phys. Rev. B 79, 085303 (2009).

[19] S. Katsumoto, Coherence and Spin Effects in Quantum Dots, J. Phys. Condens. Matter 19, 233201 (2007).

[20] A. E. Miroshnichenko, S. Flach, and Y. S. Kivshar, Fano Resonances in Nanoscale Structures, Rev. Mod. Phys. 82, 2257 (2010). 
[21] D. K. Ferry, S. M. Goodnick, and J. P. Bird, Transport in Nanostructures (Cambridge University Press, Cambridge, England, 2009).

[22] T. Morimoto, Y. Iwase, N. Aoki, T. Sasaki, Y. Ochiai, A. Shailos, J.P. Bird, M.P. Lilly, J.L. Reno, and J. A. Simmons, Nonlocal Resonant Interaction between Coupled Quantum Wires, Appl. Phys. Lett. 82, 3952 (2003).

[23] V. I. Puller, L. G. Mourokh, A. Shailos, and J. P. Bird, Detection of Local-Moment Formation Using the Resonant Interaction between Coupled Quantum Wires, Phys. Rev. Lett. 92, 096802 (2004).

[24] Y. Yoon, L. Mourokh, T. Morimoto, N. Aoki, Y. Ochiai, J.L. Reno, and J.P. Bird, Probing the Microscopic Structure of Bound States in Quantum Point Contacts, Phys. Rev. Lett. 99, 136805 (2007).

[25] Y. Yoon, M.-G. Kang, T. Morimoto, L. Mourokh, N. Aoki, J. L. Reno, J. P. Bird, and Y. Ochiai, Detector Backaction on the Self-Consistent Bound State in Quantum Point Contacts, Phys. Rev. B 79, 121304(R) (2009).

[26] Y. Yoon, M.-G. Kang, P. Ivanushkin, L. Mourokh, T. Morimoto, N. Aoki, J. L. Reno, Y. Ochiai, and J. P. Bird, Non-Local Bias Spectroscopy of the Self-Consistent Bound State in Quantum Point Contacts near Pinch-off, Appl. Phys. Lett. 94, 213103 (2009).

[27] See the special issue on The 0.7 Feature and Interactions in One-Dimensional Systems in J. Phys. Condens. Matter 20, 160301 (2008).

[28] A.P. Micolich, What Lurks below the Last Plateau: Experimental Studies of the $0.7 \times 2 e^{2} / h$ Conductance Anomaly in One-Dimensional Systems, J. Phys. Condens. Matter 23, 443201 (2011).

[29] K. Hirose, Y. Meir, and N.S. Wingreen, Local Moment Formation in Quantum Point Contacts, Phys. Rev. Lett. 90, 026804 (2003).

[30] T. Rejec and Y. Meir, Magnetic Impurity Formation in Quantum Point Contacts, Nature (London) 442, 900 (2006).

[31] A. D. Güçlü, C. J. Umrigar, H. Jiang, and H. U. Baranger, Localization in an Inhomogeneous Quantum Wire, Phys. Rev. B 80, 201302(R) (2009).

[32] E. Welander, I. I. Yakimenko, and K.-F. Berggren, Localization of Electrons and Formation of TwoDimensional Wigner Spin Lattices in a Special Cylindrical Semiconductor Stripe, Phys. Rev. B 82, 073307 (2010).

[33] T. Song and K.-H. Ahn, Ferromagnetically Coupled Magnetic Impurities in a Quantum Point Contact, Phys. Rev. Lett. 106, 057203 (2011).
[34] O.P. Sushkov, Restricted and Unrestricted Hartree-Fock Calculations of Conductance for a Quantum Point Contact, Phys. Rev. B 67, 195318 (2003).

[35] K. F. Berggren and I. I. Yakimenko, Nature of Electron States and Symmetry Breaking in Quantum Point Contacts According to the Local Spin Density Approximation, J. Phys. Condens. Matter 20, 164203 (2008).

[36] Y. Ren, W.W. Yu, S. M. Frolov, J.A. Folk, and W. Wegscheider, Zero-Bias Anomaly of Quantum Point Contacts in the Low-Conductance Limit, Phys. Rev. B 82, 045313 (2010).

[37] See Supplemental Material at http://link.aps.org/supplemental/10.1103/PhysRevX.2.021003 for a detailed description of the measurement procedures and of the theoretical derivation of our model.

[38] M. Field, C. G. Smith, M. Pepper, D. A. Ritchie, J. E. F. Frost, G. A. C. Jones, and D. G. Hasko, Measurements of Coulomb Blockade with a Noninvasive Voltage Probe, Phys. Rev. Lett. 70, 1311 (1993).

[39] J.R. Schrieffer and P. A. Wolff, Relation between the Anderson and Kondo Hamiltonians, Phys. Rev. 149, 491 (1966).

[40] J. W. Song, Y. Kawano, K. Ishibashi, J. Mikalopas, G. R. Aizin, N. Aoki, J.L. Reno, Y. Ochiai, and J.P. Bird, Current-Voltage Spectroscopy of the Subband Structure of Strongly Pinched-off Quantum Point Contacts, Appl. Phys. Lett. 95, 233115 (2009).

[41] J. J. Lin and J. P. Bird, Recent Experimental Studies of Electron Dephasing in Metal and Semiconductor Mesoscopic Structures, J. Phys. Condens. Matter 14, R501 (2002).

[42] N. J. Craig, J. M. Taylor, E. A. Lester, C. M. Marcus, M. P. Hanson, and A.C. Gossard, Tunable Nonlocal Spin Control in a Coupled-Quantum Dot System, Science 304, 565 (2004).

[43] S. Sasaki, S. Kang, K. Kitagawa, M. Yamaguchi, S. Miyashita, T. Maruyama, H. Tamura, T. Akazaki, Y. Hirayama, and H. Takayanagi, Nonlocal Control of the Kondo Effect in a Double Quantum Dot-Quantum Wire Coupled System, Phys. Rev. B 73, 161303(R) (2006).

[44] D. Tutuc, B. Popescu, D. Schuh, W. Wegscheider, and R. J. Haug, Tunable Nonlocal Coupling between Kondo Impurities, Phys. Rev. B 83, 241308(R) (2011).

[45] L. Hoshik and L. E. Reichl, Dicke Effect in a Multiripple Electron Waveguide, Phys. Rev. B 77, 205318 (2008).

[46] R.H. Dicke, Coherence in Spontaneous Radiation Processes, Phys. Rev. 93, 99 (1954).

[47] T. Brandes, Coherent and Collective Quantum Optical Effects in Mesoscopic Systems, Phys. Rep. 408, 315 (2005). 